\title{
Effect of atrial antitachycardia pacing treatments in patients with an atrial defibrillator: randomised study comparing subthreshold and nominal pacing outputs
}

\author{
A R J Mitchell, P A R Spurrell, L Cheatle, N Sulke
}

See end of article for authors' affiliations

Correspondence to:

Dr Andrew Mitchell

Department of Cardiology,

Eastbourne General

Hospital, King's Drive,

Eastbourne, East Sussex

BN2 1 2UD, UK;

mitcharj@doctors.org.uk

Accepted

29 January 2002
Heart 2002;87:433-437

\begin{abstract}
Objective: To assess the true efficacy of antitachycardia pacing on spontaneous persistent atrial fibrillation in patients with an implanted atrial defibrillator, by comparing the effects of nominal pacing treatment with subthreshold pacing treatment.

Design: The effects of antitachycardia pacing and burst $50 \mathrm{~Hz}$ atrial pacing on spontaneous atrial arrhythmias were evaluated six months after implantation of a Medtronic Jewel $\mathrm{AF}^{\oplus}$ atrial defibrillator. Setting: Cardiology department in a district general hospital.

Patients: 15 patients with persistent atrial fibrillation.

Interventions: Patients were randomised to either "nominal" output pacing treatment or surface ECG and endocardial electrogram proven subthreshold "sham" pacing treatment for three months, and then crossed over to the alternative treatment for a further three months.

Results: During the nominal output phase, 31 episodes of atrial fibrillation were treated with 53 bursts of $50 \mathrm{~Hz}$ pacing, 98 sequences of ramp atrial pacing, and 61 sequences of burst atrial pacing. Atrial fibrillation was not pace terminated during any episode. Thirty one episodes of atrial tachycardia were treated with 19 bursts of $50 \mathrm{~Hz}$ atrial pacing, 103 sequences of ramp atrial pacing, and 38 sequences of burst atrial pacing. Termination of atrial tachycardia was observed in 17 episodes. During the "sham" pacing period, no episodes were terminated by any pacing treatment.

Conclusion: Atrial antitachycardia pacing treatments are ineffective at terminating persistent atrial fibrillation but may be useful in terminating episodes of atrial tachycardia or flutter, thus reducing the burden of arrhythmia.
\end{abstract}

$\mathrm{T}$ he management of paroxysmal and persistent atrial fibrillation has changed over the last decade, with a gradual move away from reliance on medical treatment alone. Hybrid treatments involving various electrophysiological and pacing techniques are now being using in synergy with conventional pharmaceutical treatment. Newer and more sophisticated pacemaker technologies are being investigated and efforts focused on finding methods of either terminating atrial fibrillation early or reducing the burden of arrhythmia. ${ }^{1}$

The Medtronic (Minneapolis, USA) Jewel $\mathrm{AF}^{\circledR}$ defibrillator (model 7250) allows tiered atrial treatment to be delivered on detection of atrial arrhythmias. Stored Holter and endocardial electrogram strip recordings allow the precise onset and termination of atrial arrhythmias to be determined and the effects of treatment assessed. The patients who seem to benefit particularly from the atrial defibrillator are those in whom the only arrhythmia is recurrent drug resistent persistent atrial fibrillation. ${ }^{2}$ Previous studies investigating the effects of atrial pacing treatment using the Jewel $\mathrm{AF}^{\oplus}$ have, however, also included patients with paroxysmal atrial fibrillation and ventricular arrhythmias. ${ }^{3}$

Our aims were to investigate atrial pacing treatment in patients with persistent atrial fibrillation only, as follows: (1) to show the effects of atrial pacing at either nominal or subthreshold "sham" outputs on spontaneous persistent atrial fibrillation, using a randomised crossover design; (2) to investigate the efficacy of $50 \mathrm{~Hz}$ atrial pacing for terminating induced and spontaneous persistent atrial fibrillation.

\section{METHODS}

Patients

A Medtronic Jewel $\mathrm{AF}^{\circledR}$ atrial defibrillator was implanted in 15 patients, the sole indication being drug refractory persistent atrial fibrillation. The participants were 12 men and three women (mean age 63.0 years, range 38 to 83 ). None of them had a history of ventricular arrhythmia or any documented evidence of paroxysmal atrial fibrillation on repeated preimplant 24 hour Holter monitoring and careful review of the clinical records. All patients underwent cardioversion to sinus rhythm during implantation as part of the device testing procedure.

\section{Effects of $50 \mathrm{~Hz}$ atrial pacing on induced atrial fibrillation}

At predischarge testing, atrial fibrillation was induced in all patients by a two second burst of $50 \mathrm{~Hz}$ atrial pacing at an amplitude of $8 \mathrm{~V}$ and a pulse width of $1.5 \mathrm{~ms}$. Atrial fibrillation was allowed to stabilise for two minutes. A two second burst of $50 \mathrm{~Hz}$ atrial pacing was then delivered in an attempt to terminate the rhythm. If atrial fibrillation persisted, then the rhythm was finally cardioverted by patient activated defibrillation using the Jewel $\mathrm{AF}^{\oplus}$ and a remote activator (Medtronic model 9464).

\section{Effects of atrial pacing treatments on spontaneous atrial episodes}

All patients were studied six months after implantation of the atrial defibrillator. Each patient was randomised to three month periods of atrial treatments, with outputs set at either "nominal" ( $5 \mathrm{~V}$ output and $1.5 \mathrm{~ms}$ pulse width) or proven subthreshold "sham" pacing ( $1 \mathrm{~V}$ output and $0.03 \mathrm{~ms}$ pulse width). Atrial pacing was performed at sham outputs before final programming to confirm that there was no atrial capture on either the surface ECG or the endocardial electrogram. Atrial pacing treatment was initiated after two minutes of a recognised atrial arrhythmia. After three months, the patients 


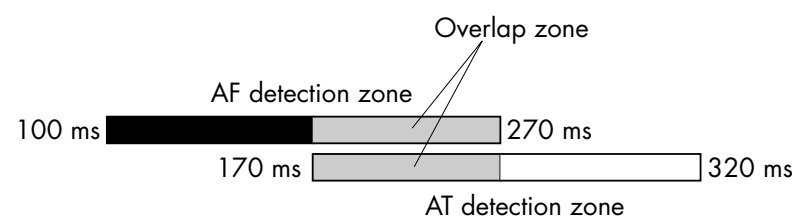

Figure 1 Classification of arrhythmia is initially dependent on the atrial cycle length. Atrial fibrillation (AF) is classified if the atrial cycle length lies between $100 \mathrm{~ms}$ and $270 \mathrm{~ms}$. Atrial tachycardia (AT) is classified if the atrial cycle length is between $170 \mathrm{~ms}$ and $320 \mathrm{~ms}$. Within the overlap zone, the classification is dependent on the regularity of the rhythm.

were crossed over to the alternative treatment. Drug treatment remained unchanged for the duration of the study.

\section{Arrhythmia classification}

The device was programmed to classify an atrial arrhythmia as atrial fibrillation if the mean atrial cycle length was between $100 \mathrm{~ms}$ and $270 \mathrm{~ms}$. Atrial tachycardia was diagnosed if the cycle length fell between $170 \mathrm{~ms}$ and $320 \mathrm{~ms}$. Within the overlap zone, the Jewel $\mathrm{AF}^{\oplus}$ discriminated between atrial fibrillation and atrial tachycardia according the regularity of the rhythm (fig 1). The rhythm was classified as regular when, in the most recent 12 intervals, the difference between the shortest and the longest atrial interval was less than or equal to $25 \%$ of the atrial median interval. Antitachycardia pacing treatment was then delivered if the cycle length was regular for six of the preceding eight intervals. If the cycle length was not stable, antitachycardia pacing treatment was withheld and counters restarted. Some tachycardia episodes could therefore be temporarily classified as different rhythms if the arrhythmia accelerated or decelerated into different detection zones or became more or less regular.

The term "paroxysmal" was used to describe any spontaneously terminating episodes of arrhythmia, whereas "persistent" was used to describe all episodes that required patient activated cardioversion. Atrial tachycardia is used to describe all regular episodes of atrial arrhythmia (including atrial flutter).

\section{Delivered treatments}

Atrial treatments were delivered according to the initial episode rhythm classification by the Jewel $\mathrm{AF}^{\oplus}$ device, and again if the arrhythmia was reclassified during each episode. On classification as atrial fibrillation, the device can only deliver $50 \mathrm{~Hz}$ atrial pacing, and a two second burst was programmed to be delivered. On classification as atrial tachycardia, the device delivered up to six sequences of atrial ramp pacing (ARamp), six sequences of atrial burst pacing (ABurst + ), and a two second burst of $50 \mathrm{~Hz}$ atrial pacing. After each delivered treatment the device looks for restoration of sinus rhythm before proceeding with the next treatment sequence (table 1). Atrial fibrillation could only receive ARamp or ABurst + treatments if the rhythm was temporarily reclassified as atrial tachycardia by the device.

\section{Atrial pacing treatment}

$50 \mathrm{~Hz}$ pacing - A two second burst of rapid atrial pacing with pacing interval of $20 \mathrm{~ms}$.

Atrial ramp pacing (ARamp)-Six pulses of AOO pacing with the first stimulus of each sequence delivered at $94 \%$ of the preceding atrial cycle length (table 1). The remainder of the sequence was delivered at progressively shorter intervals with a programmed interval decrement of $10 \mathrm{~ms}$. Each time the tachycardia was redetected the sequence was delivered at $94 \%$ of the redetected cycle length and an extra stimulus was added to the sequence.

Atrial burst pacing (ABurst+) - 10 pulses of AOO pacing at $94 \%$
Table 1 Atrial pacing treatments

\begin{tabular}{ll}
\hline AF treatments & AT treatments \\
\hline Pacing $(50 \mathrm{~Hz}$ ) & 1. Pacing (ARamp) \\
One burst, duration 2 s & Six bursts \\
& Six pulses \\
A-S1 interval 94\% of AA & Interval deceleration $10 \mathrm{~ms}$ \\
2. Pacing (ABurst+) & Six bursts \\
12 Pulses \\
A-S1 interval 94\% of AA \\
S1-S2 interval 91\% of AA \\
S2-S3 decrement 20 ms \\
Interval deceleration $10 \mathrm{~ms}$ \\
3. Pacing (50 Hz) \\
One burst, duration $2 \mathrm{~s}$ \\
\hline
\end{tabular}

For atrial fibrillation (AF), only $50 \mathrm{~Hz}$ atrial pacing can be programmed. Tiered atrial treatment can be programmed for atrial tachycardia (AT) with ramp, burst+, and $50 \mathrm{~Hz}$ pacing. Ramp and burst+ treatments can therefore only be delivered in AF if the device misclassifies the rhythms as AT.

of the atrial cycle length. This was followed by an extra stimulus at $91 \%$ of the cycle length and a further stimulus $10 \mathrm{~ms}$ shorter than $91 \%$ of the cycle length. Each time the tachycardia was redetected, the programmed intervals were reapplied with a $10 \mathrm{~ms}$ decrement to the new arrhythmia cycle length.

\section{Definition of successful termination}

Successful termination was defined as the restoration of atrioventricular synchrony for at least one beat within five atrial beats of the last stimulus of pacing treatment, even if early recurrence of the arrhythmia occurred thereafter. The Jewel $\mathrm{AF}^{\circledR}$ device, however, defines successful termination when it detects five consecutive beats of sinus rhythm within three minutes of treatment delivery. To assess the number of episodes that spontaneously terminated after pacing treatments were delivered and were therefore classified as successfully terminated by the Jewel $\mathrm{AF}^{\circledR}$, we examined endocardial electrograms of all episodes terminating within one minute of delivery of pacing treatment.

\section{Follow up}

Patients were reviewed at the end of each three month study phase, the device was interrogated using a conventional programmer, and all data were downloaded to disc. Atrial electrograms were studied and compared with the device classification of each episode. The median atrial cycle length (at the electrode tip) of each arrhythmia was recorded for each episode. After completion of the study period, atrial pacing treatments were reprogrammed at the clinician's discretion.

\section{Statistics}

Values are given as mean (SD). Atrial cycle lengths were grouped according to rhythm classification and averages and variance calculated. Comparisons of atrial cycle lengths was by Student's $t$ test. A probability ( $\mathrm{p}$ ) value of $<0.05$ indicated statistical significance.

\section{RESULTS}

\section{Spontaneous atrial episodes}

Mean follow up periods were 93.4 days with nominal output and 92.7 days with sham output. Sixty two atrial episodes occurred during the nominal period and 67 during the sham phase. Episodes were classified according to interpretation of stored atrial electrograms. There were 57 episodes of atrial fibrillation, with 11 spontaneously terminating episodes. The mean cycle length of persistent atrial fibrillation was 199 (38) $\mathrm{ms}$, and of paroxysmal atrial fibrillation, 238 (36) ms $(p<0.005)$. There were 72 episodes of atrial tachycardia, with 
Table 2 Arrhythmia episode frequency, distribution, and mean atrial cycle length $(A C L)$

\begin{tabular}{llll}
\hline & $\begin{array}{l}\text { No of } \\
\text { episodes }\end{array}$ & $\begin{array}{l}\text { No of } \\
\text { patients }\end{array}$ & Mean ACL (ms) \\
\hline Persistent AF & 46 & 10 & $199(38)$ \\
Paroxysmal AF & 11 & 3 & $238(36)$ \\
Persistent AT & 6 & 2 & $217(36)$ \\
Paroxysmal AT & 49 & 4 & $228(32)$ \\
Terminated AT & 17 & 1 & $234(33)$ \\
\hline
\end{tabular}

Values are mean (SD).

$A F$, atrial fibrillation; $A T$, atrial tachycardia.

49 episodes terminating spontaneously. There was no significant difference between the mean cycle lengths of persistent atrial tachycardia and paroxysmal atrial tachycardia: 217 (36) $v 228$ (32) ms, $\mathrm{p}=0.37$ (table 2).

The device initially classified arrhythmic episodes as atrial fibrillation in 42 cases and atrial tachycardia in 15 cases. Of these latter 15 episodes, 11 were subsequently reclassified by the device as atrial fibrillation. Overall, 49 of the 57 episodes of atrial fibrillation were temporarily classified as atrial tachycardia and atrial tachycardia treatments were given.

The effect of antitachycardia pacing treatment Delivered treatments are documented in table 3. "ARamp" treatments were delivered during 58 atrial episodes when programmed to nominal output with 201 sequences of pacing treatment. During the sham period, 63 episodes received 292 sequences of treatment. "ARamp" antitachycardia pacing failed to terminate a single case of atrial fibrillation; however, it successfully terminated 16 episodes of atrial tachycardia in one patient (mean of two sequences of treatment). "Burst + " pacing was delivered 99 times during 25 episodes with nominal outputs, and 204 times in 36 episodes when programmed to sham treatment. It was ineffective at terminating any episode of atrial fibrillation but was successful in one episode of atrial tachycardia at nominal outputs (one sequence). The mean atrial cycle length of terminated atrial tachycardia (234 (33) ms) was not significantly different from either persistent tachycardia $(217(36) \mathrm{ms}, \mathrm{p}=0.31)$ or paroxysmal tachycardia (228 (32) ms, p = 0.24). Sham pacing failed to terminate any arrhythmia episode.

\section{The effect of $50 \mathrm{~Hz}$ atrial pacing}

Atrial fibrillation

Thirty one episodes of atrial fibrillation were treated with 50 $\mathrm{Hz}$ pacing with outputs programmed to nominal and 26 to sham. A further burst of $50 \mathrm{~Hz}$ was delivered in 22 episodes of atrial fibrillation with nominal outputs and 21 with sham pacing. The $50 \mathrm{~Hz}$ pacing failed to terminate any episode of atrial fibrillation, at either nominal or sham outputs.

\section{Atrial tachycardia}

Thirteen episodes of atrial tachycardia were treated with $50 \mathrm{~Hz}$ atrial pacing with nominal outputs and 13 with sham pacing.
A further burst of $50 \mathrm{~Hz}$ atrial pacing was delivered in six episodes of atrial tachycardia with nominal outputs and in 20 episodes with sham outputs. The $50 \mathrm{~Hz}$ pacing was unsuccessful at terminating any of these episodes.

\section{"Delayed termination"}

The stored endocardial electrogram and timed marker channel traces for each spontaneously terminating arrhythmia episode were examined and the time between the last pacing treatment and the end of the arrhythmia was noted. Five episodes terminated within 10 seconds of the last pacing treatment (two during nominal outputs and three during sham). Additionally, three episodes terminated within 30 seconds of the last delivered treatment (all during the sham phase), and four further episodes terminated within one minute of the delivered treatment (one during nominal pacing and three during sham pacing). All these episodes were atrial tachycardia.

\section{Induced atrial fibrillation}

Prehospital discharge testing occurred within one week of implantation. The effect of $50 \mathrm{~Hz}$ atrial pacing was evaluated in all 15 patients, as described above, and was ineffective at terminating induced atrial fibrillation in any case.

\section{DISCUSSION}

Our study showed that both $50 \mathrm{~Hz}$ atrial pacing and specific atrial antitachycardia pacing failed to terminate persistent atrial fibrillation, but ramp pacing had some efficacy in treating spontaneous onset paroxysmal atrial flutter or tachycardia. Pace termination of atrial fibrillation has not, to date, been shown to be possible in the laboratory setting. Allessie and coworkers found that during induced atrial fibrillation in the dog it was possible to capture small regions of atrial tissue by rapid pacing, ${ }^{56}$ particularly when pacing the left atrium. They found that pacing with a cycle length slightly shorter than the mean fibrillation interval led to the penetration of paced wavefronts into the excitable gap. Daoud et al similarly demonstrated regional capture during pacing induced atrial fibrillation, with acceleration and increased disorganisation in distant atrial electrograms, but failed to terminate the rhythm. ${ }^{7}$

Atrial flutter, on the other hand, is well known to be amenable to pace termination. In 1977, Waldo et al showed entrainment and pace termination of atrial flutter. ${ }^{8}$ Termination of atrial fibrillation is more difficult owing to the inhomogeneity of the atrial tissue, the numbers and variable size of separate wandering wavelets, and the smaller excitable gap. In addition, when Allessie et al paced atrial fibrillation faster than its cycle length, instead of termination of the arrhythmia (as might be seen with atrial flutter), the rhythm accelerated, resulting in loss of local capture. ${ }^{5}$

A significant proportion of cases of atrial fibrillation may start with atrial flutter or atrial tachycardia and degenerate into atrial fibrillation. ${ }^{9}{ }^{10}$ This allows a window of opportunity to pace-terminate such arrhythmias. Previous studies of the

Table 3 Number of atrial treatments delivered with pacemaker outputs programmed to nominal or sham

\begin{tabular}{|c|c|c|c|c|c|c|c|c|}
\hline & & $\begin{array}{l}\text { No of } \\
\text { episodes }\end{array}$ & $\begin{array}{l}\text { AF treatment 1: } \\
\text { AF } 50 \mathrm{~Hz}\end{array}$ & $\begin{array}{l}\text { AT treatment 1: } \\
\text { ARamp }\end{array}$ & $\begin{array}{l}\text { No of } \\
\text { sequences }\end{array}$ & $\begin{array}{l}\text { AT treatment 2: } \\
\text { ABurst+ }\end{array}$ & $\begin{array}{l}\text { No of } \\
\text { sequences }\end{array}$ & $\begin{array}{l}\text { AT treatment 3: } \\
50 \mathrm{~Hz}\end{array}$ \\
\hline \multirow[t]{3}{*}{ Nominal } & $\mathrm{AF}$ & 31 & 31 & 29 & 98 & 17 & 61 & 22 \\
\hline & Flutter & 31 & 13 & 29 & 103 & 8 & 38 & 6 \\
\hline & Total & 62 & 44 & 58 & 201 & 25 & 99 & 26 \\
\hline & $\mathrm{AF}$ & 26 & 26 & 22 & 108 & 15 & 87 & 21 \\
\hline \multirow{2}{*}{ (subthreshold) } & Flutter & 41 & 13 & 41 & 184 & 21 & 117 & 20 \\
\hline & Total & 67 & 37 & 63 & 292 & 36 & 204 & 41 \\
\hline
\end{tabular}

AF, atrial fibrillation; AT, atrial tachycardia. 


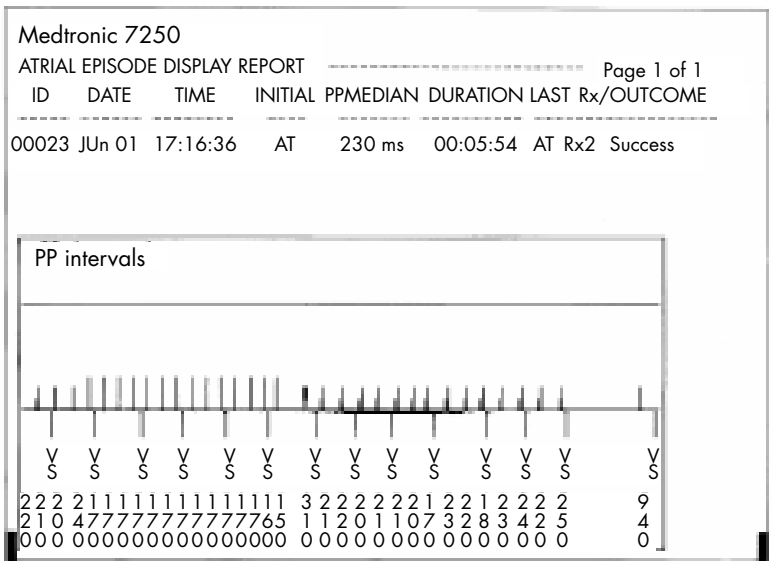

Figure 2 An episode of atrial tachycardia terminating 15 atrial beats after the delivery of subthreshold "sham" pacing The event markers show atrial sensed events above the line with ventricular sensed events below. The numbers indicate the $p-p$ intervals. After a sequence of burst+ antitachycardia pacing at subthreshold outputs, the episode of atrial tachycardia terminates after a further 15 atrial beats.

atrial defibrillator have suggested that $50 \mathrm{~Hz}$ atrial burst pacing and atrial antitachycardia pacing can terminate a significant proportion of atrial arrhythmias. ${ }^{3411-14}$ The effects of pacing treatments in patients with paroxysmal atrial fibrillation only have been evaluated in a pacemaker arrhythmia management device, the Medtronic AT500. Only 75 of 322 atrial fibrillation episodes $(23 \%)$ were terminated by antitachycardia pacing treatments. ${ }^{15}$

All such termination studies have, however, included a large number of patients with either paroxysmal atrial fibrillation or ventricular arrhythmias. They have also relied on device classification of the arrhythmia type, and on the device to determine whether treatment has been successful. The Jewel $\mathrm{AF}^{\oplus}$ defines success when it detects five beats of sinus rhythm within three minutes of treatment delivery. In our study, a small number of tachycardia episodes terminated spontaneously within one minute of the delivery of antitachycardia pacing treatments-three within the nominal phase and nine in the sham phase. Figure 2 shows one such episode, which was classified as successfully terminated by the device. In the same patient, however, when pace termination occurred with nominal outputs, episodes terminated immediately after pacing treatments (fig 3). As the Jewel $\mathrm{AF}^{\circledR}$ classifies success within three minutes of antitachycardia pacing treatment, there are of course episodes that terminate spontaneously within this period and are then erroneously classified as successfully terminated by the device.
This highlights the problem of defining successful termination. We have used an immediate restoration of sinus rhythm to define success, although it is clear from our data that if we had used longer time periods after pacing treatments then we would have had different results. Termination within 30 seconds to one minute after treatment may be a chance finding, but if termination occurs within a few seconds of treatment it is likely to be a therapeutic effect. Twenty seven of 129 episodes were incorrectly classified as "successfully terminated" by the device in this study, although none of those episodes was atrial fibrillation and they were mostly confined to one patient. Each episode's electrogram and marker traces were critically reviewed before success was defined in our study. Many studies investigating device treatment for termination of atrial fibrillation need to be interpreted with caution when using device defined criteria alone.

High frequency $(50 \mathrm{~Hz})$ burst atrial pacing is often used to initiate atrial fibrillation during electrophysiological studies, and for testing atrial defibrillators. The failure of $50 \mathrm{~Hz}$ burst pacing to terminate atrial fibrillation in this study is in keeping with several studies of induced atrial fibrillation in man. Paladino and coworkers induced atrial fibrillation in 28 patients and attempted high frequency pacing from single and multiple sites, with no terminations. ${ }^{16}$ Similar work with varying burst durations of $50 \mathrm{~Hz}$ atrial pacing revealed termination in six of 10 patients with atypical atrial flutter but no success in any patients with atrial fibrillation. ${ }^{17}$

Our study investigated the effects of atrial treatments in a specific group of patients with persistent atrial fibrillation only. There was no previous history of spontaneously terminating episodes in spite of multiple Holter tests, and no history of ventricular arrhythmias. However, four patients had self terminating episodes of atrial fibrillation or atrial tachycardia during the study period. In one of these patients and during nominal outputs, 17 of 25 episodes of atrial tachycardia were pace terminated by ramp atrial pacing. It seems that the early termination of these episodes did not result in a reduced requirement for defibrillation, because in the same patient all atrial tachycardia episodes terminated spontaneously during the sham phase of the study, without requiring defibrillation.

\section{Study limitations}

The main aim of our study was to investigate the effects of pacing treatments on spontaneous persistent atrial fibrillation. We allowed a two minute period for the rhythm to stabilise before starting pacing treatment, to ensure that there was no early spontaneous termination. It may be that during that period some arrhythmia episodes are more amenable to pace termination. Similarly, some episodes of persistent atrial

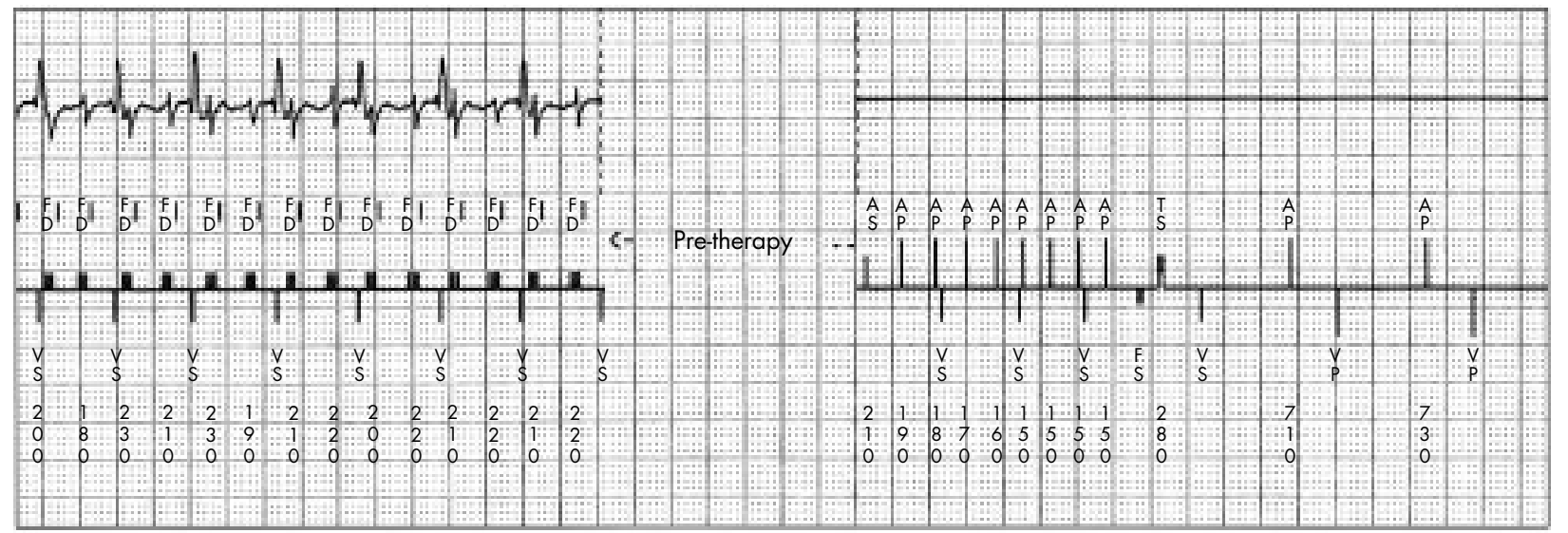

Figure 3 An episode of atrial tachycardia terminating after a sequence of ramp pacing at nominal outputs. 
fibrillation may have begun with a more regular rhythm, such as atrial flutter, which could have been pace terminated within this two minute period.

We programmed our pacing treatments according to the manufacturer's instructions, and these settings may not necessarily be optimal for the entire group of patients. Close examination of stored electrogram strips could allow pacing treatments to be programmed more accurately for each patient.

Finally, eliminating the factory default overlap zone between atrial fibrillation and atrial tachycardia by programming a large atrial tachycardia zone would have ensured that all episodes of atrial fibrillation received antitachycardia pacing treatment.

\section{Conclusions}

We have demonstrated an inability to pace terminate persistent atrial fibrillation using $50 \mathrm{~Hz}$ atrial pacing and specific antitachycardia pacing algorithms. We have, however, shown that even in a highly selected group of patients with no preimplant history of atrial tachycardia or paroxysmal atrial fibrillation, a small number of such episodes occurred and could be pace terminated. These features should therefore be made available in future devices to decrease the arrhythmia burden.

\section{Authors' affiliations}

A R J Mitchell, P A R Spurrell, N Sulke, Department of Cardiology, Eastbourne General Hospital, Eastbourne, East Sussex, UK L Cheatle, Medtronic (UK) Ltd, Watford, Hertfordshire, UK

\section{REFERENCES}

1 Kamalvand $\mathbf{K}$, Sulke N. Recent advances in the non-surgical management of atrial fibrillation. Int J Clin Pract 1999;53:44-9.

2 Josephson ME. New approaches to the management of atrial fibrillation. The role of the atrial defibrillator. Circulation 1998;98:1594-6.

3 Sulke N, Bailin SJ, Swedlow CD. Worldwide clinical experience with a dual-chamber implantable cardioverter defibrillator in patients with atrial fibrillation and flutter [abstract]. Eur Heart J 1999;20:1 14.
4 Ricci R, Altamura G, Pandozi C, et al. Efficacy of anti-tachy-pacing and low energy cardioversion in terminating spontaneous atrial tachyarrhythmias in patients with dual-chamber implantable defibrillator [abstract]. Eur Heart J 1999;20:115.

5 Allessie M, Kirchhof C, Scheffer GJ, et al. Regional control of atrial fibrillation by rapid pacing in conscious dogs. Circulation 1991;84: 1689-97

6 Kirchhof C, Chorro F, Scheffer GJ, et al. Regional entrainment of atrial fibrillation studied by high-resolution mapping in open-chest dogs. Circulation 1993;88:736-49.

7 Daoud EG, Pariseau B, Niebauer M, et al. Response of type 1 atrial fibrillation to atrial pacing in humans. Circulation 1996;94:1036-40.

8 Waldo AL, Mclean WA, Karp RB, et al. Entrainment and interruption of atrial flutter with atrial pacing therapies: studies in man following open heart surgery. Circulation 1977;56:737-45

9 Lerman B, Stein K, Markowitz S. Worldwide clinical experience with the model 7250 Jewel AF dual chamber arrhythmia management device [abstract]. Pacing Clin Electrophysiol 1998:21:872.

10 Wolpert C, Jung W, Schimpf R, et al. The incidence of atrial tachyarrhythmias during long-term follow-up of patients with a dual-chamber defibrillator supports the use of atrial antitachycardia pacing therapies [abstract]. Eur Heart J 2000;21:469.

11 Ramekan M, Schwacke H, Siemon G, et al. Atrial tachyarrhythmias in patients with a dual-chamber cardioverter-defibrillator: efficacy of atrial therapies [abstract]. J Am Coll Cardiol 2000;35:105.

12 Gold MR, Daubert JP, Peters RW, et al. A clinical trial of a combined atrial and ventricular defibrillator in patients without ventricular arrhythmias [abstract]. J Am Coll Cardiol 2000;35:115.

13 Santini M, Ricci R, Capucci A, et al. Efficacy of anti-tachy-pacing and low energy cardioversion in terminating spontaneous atrial tachyarrhythmias is patients with dual implantable defibrillator [abstract]. Eur Heart J 2000;21:470.

14 Ricci R, Pandozi C, Altamura G, et al. Efficacy of anti-tachy-pacing and low energy cardioversion in terminating spontaneous atrial tachyarrhythmias in patients with dual-chamber implantable defibrillator [abstract]. J Am Coll Cardiol 2000;35:104.

15 Huegl B, Ziegenbalg K, Doll N, et al. Is overdrive stimulation therapy a therapeutic option in pacemaker patients with paroxysmal atrial fibrillation? First results of a new dual chamber stimulator [abstract]. Eur Heart J 2000;21;604.

16 Paladino W, Bahu M, Knight B, et al. Failure of single and multisite high-frequency atrial pacing to terminate atrial fibrillation. Am J Cardiol 1997;80:226-7.

17 Giorgberidze I, Saksena S, Mongeon L, et al. Effects of high-frequency atrial pacing in atypical atrial flutter and atrial fibrillation. J Intervent Cardiac Electrophysiol 1997;1:111-23.

\section{IMAGES IN CARDIOLOGY}

\section{Magnetic resonance angiography showing bilateral subclavian artery aneurysm and stenosis in Marfan's syndrome}

$\Lambda^{26}$

26 year old woman with Marfan's syndrome presented with right sided neck discomfort and buzzing, but without neurological symptoms. A pulsatile swelling was palpable above the right clavicle.

The patient had previously had a dissecting aneurysm of the ascending aorta at the age of 15 years, for which her aortic valve and ascending aorta had been replaced with a Bjork-Shiley prosthetic valve and Dacron graft. At the age of 19 she had graft replacement of her descending thoracic aorta, distal to the left subclavian artery, for a further dissecting aneurysm.

Gadolinium enhanced magnetic resonance angiography showed localised fusiform aneurysms, maximum diameter $23 \mathrm{~mm}$, close to the origins of both right and left subclavian arteries (right). There was also evidence of stenosis of both right and left subclavian arteries distal to each aneurysm, the left sided stenosis being more severe than that on the right. Both vertebral arteries originated from the aneurysmal subclavian artery segments. No dissection of the aortic arch or its branches was seen.

This unusual pattern of arteriopathy, apparently associated with Marfan's disease, with almost symmetric dilatation and stenosis of both subclavian arteries, has not, as far as we know, been reported previously.

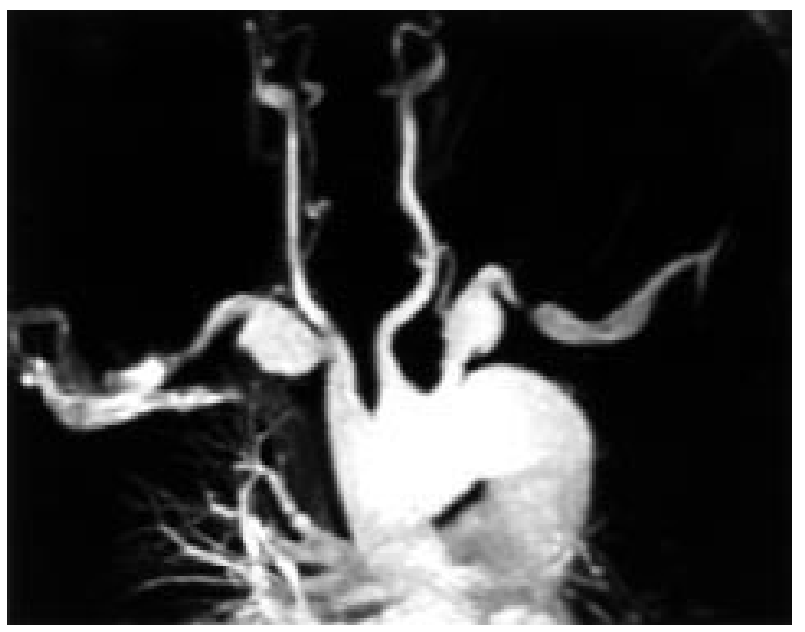

